\title{
Another Space-Filling Trefoil Knot
}

\section{P. Schmitt}

Institut für Mathematik, Universität Wien, Strudlhofgasse 4, A-1090 Wien, Austria schmitt@awirap.bitnet

\begin{abstract}
This note describes a toroidal polyhedron which is homotopic to a trefoil knot and admits an isohedral tiling of 3-space with many symmetries.
\end{abstract}

\section{Introduction}

This note describes in detail a space-filling "knotted" polyhedron (corresponding to a trefoil knot) that admits a tiling of 3-space. It is a sequal to [4], and describes the polyhedron mentioned in the remark at the end of that paper. This second example of a space-filling trefoil knot differs from the previous one in that it admits an isohedral (i.e., tile-transitive) tiling that possesses more symmetries, namely, all the direct symmetries of the cube lattice. Furthermore, the tiles of this new tiling are more intricately linked with each other.

The idea on which both the construction below and the previous construction are based can be generalized and used to obtain space-filling knotted polyhedra corresponding to arbitrary types of knots. This generalization has been briefly discussed in [5], and will be described in more detail in a forthcoming paper.

These investigations were initiated by a lecture of Schulte [6] during which he suggested searching for a space-filling knotted polyhedron. A different construction of space-filling knots is given by Kuperberg [2]. His knotted polyhedra admit a lattice tiling of 3-space, without interlocking. Furthermore, another example of a spacefilling trefoil knot was constructed earlier by McMullen [3]. Independently, the problem was also recently studied by Colin Adams.

\section{The Construction of a Knotted Space Filler: A Trefoil Knot}

Step 1. The construction starts with a regular tiling of 3-space by congruent cubes. Assume that the cubes have sides of length 1 , and that the centers are at the integer 
lattice points: Let $S_{1}$ be the union of all cubes for which the center point has (at least) two even coordinates, and let $S_{2}$ be the union of all cubes for which the center points have (at least) two odd coordinates. Then $S_{1}$ and $S_{2}$ are congruent, have disjoint interiors, and their union covers the space. Furthermore, these two sets are linked like (in 3-space) the graph formed by the edges of a regular cube tiling and the (congruent) graph obtained by joining each center of a cube with the centers of the six cubes adjacent to it. (Remark: The same decomposition of 3-space was used by Debrunner [1].)

Step 2. Let $\mathscr{T}_{1}$ be the cube tiling determined by lattice points of the form $(6 k, 6 m, 6 n)$ $\left(k, m, n\right.$ are integers) as vertices, and let $\mathscr{T}_{2}$ be $\mathscr{T}_{1}$ shifted by $(3,3,3)$. Denote by $\mathscr{T}_{i} \mid S_{i}:=\left\{T \cap S_{i} \mid T \in \mathscr{F}_{i}\right\}$ the tiling of $S_{i}$ induced by $\mathscr{T}_{i}$. Then $\left(\mathscr{T}_{1} \mid S_{1}\right) \cup$ $\left(\mathscr{T}_{2} \mid S_{2}\right)$ is a tiling by congruent tiles that still has the symmetries of a cubic lattice. The corresponding prototile $P$ is a cube $C$ of side length 6 , through which 27 holes in the form of a quadratic prism (side length 1) have been drilled $-3 \times 3$ parallel to each of the three edge directions. Its structure - with respect to connectivity - corresponds to the structure of the edge graph of a composite cube formed by $3 \times 3 \times 3$ cubes.

Step 3. As is described in detail below, the prototile $P$, obtained in the previous step, can be dissected into eight identical pieces, each directly congruent to a "knotted polyhedron" $K$, i.e., a polyhedron which is homeomorphic to a torus, and which is homotopic to a trefoil knot. This polyhedron $K$ is the desired knotted space filler: Eight copies of $K$ tile $P$, and $P$ is a space filler. Therefore $K$ is a space filler.

The dissection of $P$ into eight copies of $K$ preserves the direct symmetries of the basic cube $C$. In particular, $K$ possesses threefold rotational symmetry-one copy of it is situated "around" each vertex of $C$ (see Fig. 4 which shows a simplified knot embedded in the cube). $P$ can be considered as the union of six identical square "pyramids" (more precisely, square pyramids with holes) determined by the center and one of the six "sides" of $P$ (or $C$ ). Due to its symmetry, the dissection of $P$ induces identical dissections of these pyramids into four congruent parts, each corresponding to one-third of a copy of $K$, and consisting of two (noncongruent) pieces. Moreover, this dissection preserves the fourfold rotational symmetry of the square pyramids, and each knot passes through three of these pyramids (namely, those sharing a vertex of $C$ ). Therefore, in order to describe the polyhedron $K$, and how it tiles $P$, it is sufficient to describe how one-third of $K$ is embedded into a pyramid.

Figure 1 shows such a generating pyramid $Q$ in a side view, together with three cross sections (taken parallel to the.base of the pyramid, i.e., a side of the cube), as they are used to describe the knot. (The three marks at the left of the side view show where the sections are taken. It is assumed that these sections are taken slightly higher than the corresponding horizontal sides.)

Figure 2 describes one-third of $K$, and how it lies in the generating pyramid. It shows the three cross sections. The parts shown in black indicate that the corresponding part of the pyramid $Q$ above this region (up to the next cross section) belongs to $K$. Note that this describes a set $L$ consisting of two connected 

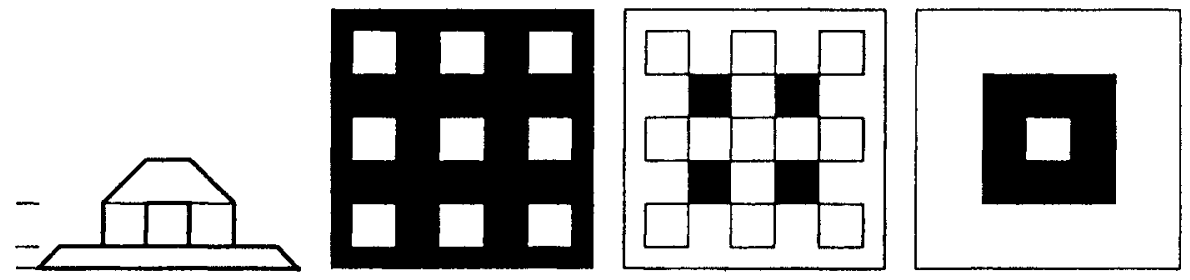

Fig. 1. The generating pyramid.

components. Together with its images under rotation by $\pi / 2, \pi$, and $3 \pi / 2$ it forms a partition of the pyramid $Q$.

In the figure the lowest vertex of each cross section corresponds to a point on the (threefold) symmetry axis of $K$, i.e., a space diagonal of $C$. The union of $L$ and its images under rotation around this axis (by $2 \pi / 3$ and $4 \pi / 3$ ) is a copy of $K$.

Furthermore, Fig. 3 shows views (seen parallel to the base) of the four triangular sides of a generating pyramid $Q$, arranged as in a net. The shaded parts lie below the face-they are visible only because of one of the prismatic holes. The two lower

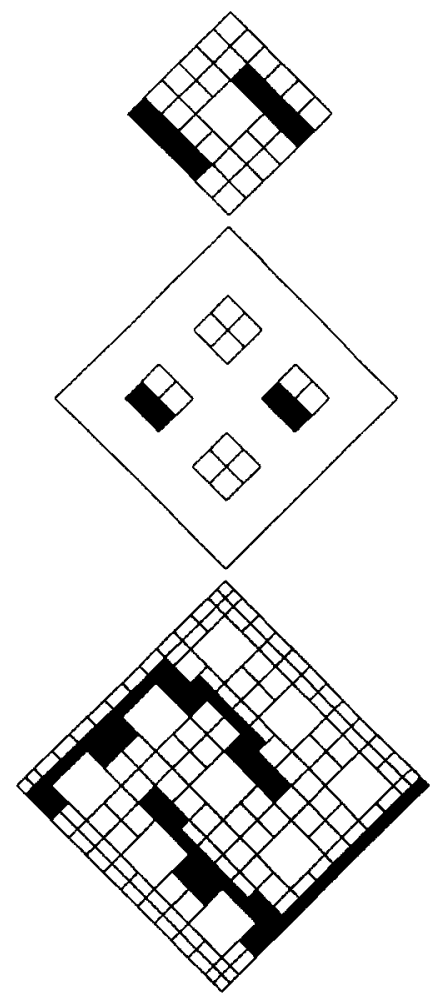

Fig. 2. Composition of the knotted tile: cross sections. 


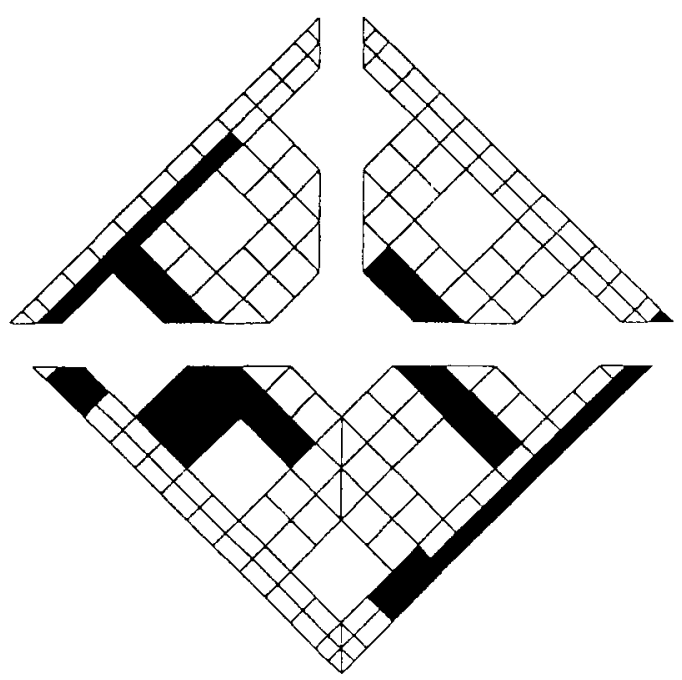

Fig. 3. Composition of the knotted tile: the triangular faces.

triangles meet in the threefold symmetry axis. When $K$ is generated by adding two rotated copies, the lower left face is placed against a copy of the lower right face, and vice versa. Thus it can be seen how the two components $L_{1}$ and $L_{2}$ of $L$ in one copy of $Q$ are connected with those in the adjacent copies $\left(Q^{\prime}\right.$ and $\left.Q^{\prime \prime}\right)$ of $Q-L_{1}$ to $L_{2}^{\prime}$ and $L_{2}^{\prime \prime}$, and $L_{2}$ to $L_{1}^{\prime}$ and $L_{1}^{\prime \prime}$ - to form the connected polyhedron $K$, composed of (in circular sequence) $L_{1}, L_{2}^{\prime}, L_{1}^{\prime \prime}, L_{2}, L_{1}^{\prime}$, and $L_{2}^{\prime \prime}$.

Figure 4 shows how $K$-or, rather, a knot homotopic to it - is embedded into the cube $C$. The diagram is seen in the direction of a space diagonal of the cube, and

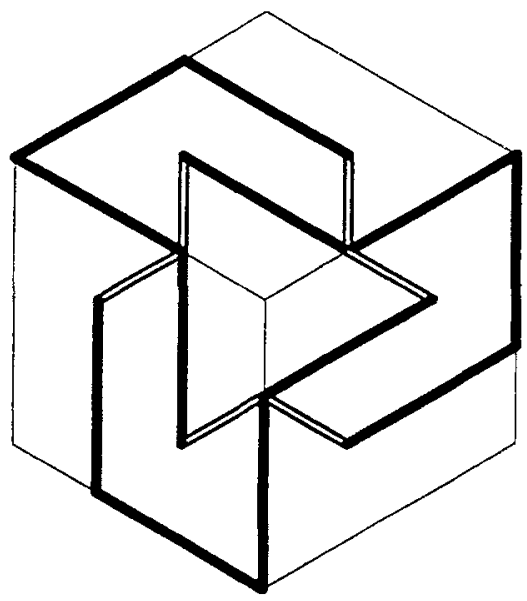

Fig. 4. The embedding of the trefoil knot. 
thus preserves the threefold symmetry. Those parts of the knot that "lie on the surface" are shown with a bold line. Parts shown with a double line lie in the interior and are line segments perpendicular to one side of the cube.

\section{References}

1. H. E. Debrunner, Tiling three-space with handlebodies. Studia Sci. Math. Hungar. 21 (1986), 201-202.

2. W. Kuperberg, Knotted lattice-like space fillers, Discrete Comput. Geom., this issue, pp. 561-567.

3. P. McMullen, personal communication.

4. P. Schmitt, A spacefilling cloverleaf knot. Note, June 14, 1993 (to be published).

5. P. Schmitt, Spacefilling knots. Preprint, 1994.

6. E. Schulte, Spacefillers of higher genus. Lecture, Austrian Conference on Discrete Geometry, May 2-8, 1993, Neuhofen/Ybbs, Austria.

Received January 26, 1994, and in revised form August 28, 1994. 